\title{
Parameterization of Submesoscale Eddy-Rich Flows Using a Stochastic Velocity Model
}

\author{
Mine ÇAĞLAR \\ Department of Mathematics, Koç University, Istanbul, Turkey \\ TAMAY M. ÖZGÖKMEN \\ RSMAS/MPO, University of Miami, Miami, Florida \\ LEONID I. PITERBARG \\ Department of Mathematics, University of Southern California, Los Angeles, California
}

(Manuscript received 20 May 2005, in final form 13 April 2006)

\begin{abstract}
In light of the recent high-resolution radar data of surface velocity, which have revealed submesoscale eddies between the Florida Current and the coast, an objective method of detecting eddies and estimating their parameters such as center coordinates, size, and intensity is suggested. The obtained statistics are used to parametrically represent the birth-death process of eddies filling up the observation area via a model stochastic velocity field known as Çinlar flow. It appears that the suggested approach leads to a reasonable parameterization of this process for potential future use in OGCMs or coastal models.
\end{abstract}

\section{Introduction}

It is believed that eddy dynamics play a key role in understanding different physical regimes of turbulent flows such as energy and enstrophy cascade (Schram et al. 2004). Ocean general circulation models (OGCMs) used to better understand large-scale ocean and climate dynamics capture only part of the turbulent cascade, as the grid scale is typically $100 \mathrm{~km}$ in climate and $10-20$ $\mathrm{km}$ in ocean process models in present-day studies. The processes that are smaller than these grid spacings, usually considered to be in the so-called submesoscale range, are typically represented by Laplacian or biharmonic eddy-diffusion operators. The understanding and representation of submesoscale phenomena in OGCMs is also limited by the sparseness of observational data in time and space. Traditional oceanobserving systems such as current meters, XBTs, and drifters provide only pointwise or one-dimensional

Corresponding author address: Tamay M. Özgökmen, Rosenstiel School of Marine and Atmospheric Science, MPO Division, University of Miami, 4600 Rickenbacker Causeway, Miami, FL 33149-1098.

E-mail: tozgokmen@rsmas.miami.edu data, and observations conducted by one or several research vessels may lead to nonsynoptic data, leading to errors resulting from interpolations in time and/or space.

The present study is motivated by two recent developments, first in observational technology and second in the theory of stochastic modeling. The first is the availability of high-frequency (HF) radar, which allows for the measurement of surface currents with unsurpassed space and time sampling. Shay et al. (2000) obtained observations of the Florida Current with a space interval of $125 \mathrm{~m}$ and time interval of $15 \mathrm{~min}$ simultaneously in a domain of $11.25 \mathrm{~km}$ by $11.25 \mathrm{~km}$. These observations revealed an episodic passage of eddies with a diameter of only several kilometers. These eddies could be related to the upwelling on cold water on the Florida shelf and, given their size and time scales, would require ultra-high-resolution nonhydrostatic models. Since small eddies as detected by HF radar measurements cannot be resolved by OGCMs, they should be parameterized for such models. A parametric stochastic model for eddy-rich flows has been recently developed by E. Çinlar (1993, 1994, personal communications) and its properties have been investigated in detail by Çağlar $(2000,2003)$ and Çağlar and Çinlar (2001). 
The mentioned observational dataset from HF radar has been previously used to derive boundary conditions for OGCMs by compressing the data into a time series of vorticity, and representing this time series in the form of a second-order autoregressive process (Mariano et al. 2003).

The aim of the present study is twofold: first, to carry out a statistical analysis of the real eddy field variability based on a relatively new method of detecting and estimating eddies suggested here and, second, to explore how well one can approximate the kinematics of oceanic eddies using the stochastic velocity model developed by Çağlar and Çinlar (2001).

Our main finding is that the submesoscale eddy variability is reasonably parameterized by that stochastic model. At the same time there is an obvious discrepancy between eddy exponential decay stated by the model and data. Thus, further extension of Çinlar flow is needed to cover different regimes in the eddy death process.

The paper is organized as follows. HF radar data are summarized in the next section, while the stochastic model is described in section 3. The methodology for estimating the parameters of the stochastic model from observational data is described in section 4 . The main results of parameter estimation and validation of the stochastic model are discussed in section 5. Finally, the most important conclusions are given in section 6 .

\section{HF radar data in the Florida Current}

To present our detection/estimation/validation methodology we employ recent observations along the Florida coast obtained by a high-frequency (HF) radar (Shay et al., 2000). These measurements cover the region bounded by $11.25 \mathrm{~km} \times 11.25 \mathrm{~km}$ during 28 days. By using a radar capable of resolving scales of $250 \mathrm{~m}$ in space and $20 \mathrm{~min}$ in time (the data have been subsequently interpolated to a spatial resolution of $125 \mathrm{~m}$ by Mariano), submesoscale cyclonic vortices of diameter of 1-3 km have been detected between the coastline and the Florida Current. These eddies transport vorticities of approximately $4 f$ (where $f$ is the local Coriolis frequency) with a speed of about $30 \mathrm{~cm} \mathrm{~s}^{-1}$ northward along the Florida Current. The reasons for the existence of these vortices are not known accurately yet, but several possible explanations have been discussed in Peters et al. (2002), such as semidiurnal tides, barotropic instability of the zonally sheared Florida Current, vorticity waves, and vortex stretching due to upwelling of denser water masses. Also, these eddies are not observed continuously, but appear to occur rather sporadically. Further details on these observations are (a)
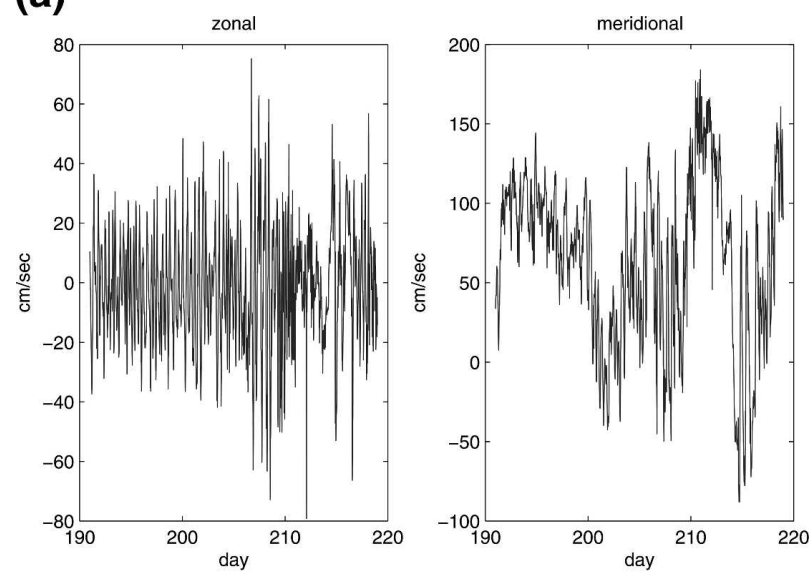

(b)
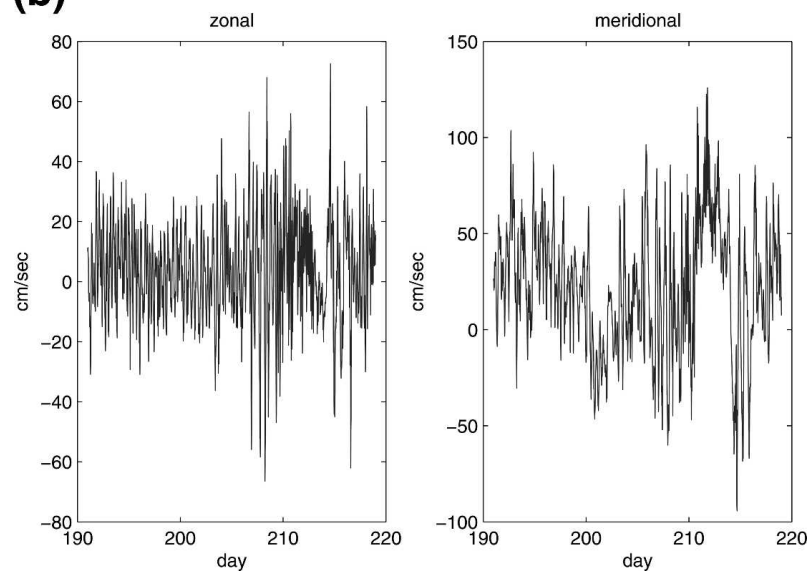

FIG. 1. (a) Velocity time series from HF radar data at the grid point $26.055^{\circ} \mathrm{N}, 80.0650^{\circ} \mathrm{W}$. (b) Velocity time series from $\mathrm{HF}$ radar data at the grid point $26.07^{\circ} \mathrm{N}, 80.08^{\circ} \mathrm{W}$.

also given in Shay et al. (2002) and Soloviev et al. (2003a,b).

The time series of the velocity components at a central location in the observation grid is given in Fig. 1. Observations at the other grid points are similar. To obtain a zero mean velocity field, the mean velocity obtained from the whole time series is subtracted from the velocity, respectively, at each grid point. Then, we apply the method described in section 4 to obtain the eddy parameters.

\section{A stochastic model for eddy-rich flow fields}

Here we briefly present a stochastic velocity model for eddy-rich flows as introduced by E. Çinlar (1993, 1994, personal communications). The model, being first-order Markov, encompasses a whole new class of random velocity fields for modeling turbulent flows. Such velocity fields are functional versions of Poisson 
shot-noise and are close to those used in vortex methods. In addition, they have other attractive features: they are stationary and ergodic and can be made homogeneous, incompressible, and isotropic easily. Therefore, the Çinlar model is better suited to capture the medium-scale coherent structures, or eddies observed in real oceanic flows, as opposed to the smallscale structures modeled by Brownian flows (Zirbel and Çinlar 1996).

The specific structures that comprise the velocity field are eddies of random size, amplitude, and position. To give a formal description of the stochastic velocity field, first introduce so-called basic eddy $V_{0}=$ $\left(u_{0}, v_{0}\right)$ defined by

$$
u_{0}(x, y)=\frac{y}{r} f(r), v_{0}(x, y)=-\frac{x}{r} f(r),
$$

where $f(r)$ is a prescribed decaying function of the distance $r$ from the origin to $(x, y)$ negligibly small as $r>$ $r_{0}$. Hence, the basic eddy vanishes outside the disk of radius $r_{0}$

The underlying velocity field is defined as a sum of random Poisson number $N$ of eddies obtained by random scattering, amplification, and dilation of the basic eddy. Namely,

$$
V(\mathbf{r}, t)=\sum_{i=1}^{N} e^{-c\left(t-s_{i}\right)} a_{i} V_{0}\left(\frac{\mathbf{r}-\mathbf{z}_{i}}{b_{i}}\right),
$$

where $\mathbf{r}=(x, y), s_{i}$ are random moments of eddy arrival (birth) forming a Poisson process in time, $c>0$ is a nonrandom decay parameter, $\mathbf{z}_{i}$ are the eddy centers, $a_{i}$ are random amplitudes, and $b_{i}$ are the random dilations.

Under broad assumptions on joint distribution of the random parameters, this velocity field is a stationary (first order) Markov process in time (see details in Çă̆lar and Çinlar 2001).

\section{Estimation method for eddy parameters}

It is important to have reliable means for detecting individual eddies and estimating the key parameters such as the center coordinates, size, and intensity (amplitude). Subsequently, other important quantities can be evaluated from a velocity field snapshot, such as the number of eddies in a given area at a given time. If a time series of such snapshots is available, then the dependence of the number of eddies on time and the individual eddy lifetime can be estimated as well. Although in many cases one can easily visualize an eddy and its trajectory when having consecutive snapshots, it is really hard to find a robust objective numerical procedure detecting individual eddies. Various approaches exist in the literature (Benzi et al. 1988; McWilliams 1990; Pasquero and Provenzale 2002; Schram et al. 2004; Guan et al. 2003; Siegel and Weiss 1997). A basic idea of our approach is to combine a nonparametric method for detecting eddies and a parametric algorithm hinted by the Çinlar model for estimating their parameters. The method for estimating eddy parameter is described in the following.

Assume that a 2D velocity field is given in some region by its components $u(x, y)$ and $v(x, y)$. The first step is to separate the velocity field in solenoidal and potential components, which is done by standard means. Namely, we first estimate the vorticity field

$$
\xi(x, y)=\frac{\partial v}{\partial x}-\frac{\partial u}{\partial y},
$$

and find the streamfunction $\psi$ by solving the equation

$$
\Delta \psi(x, y)=\xi(x, y),
$$

where $\Delta$ is the Laplacian operator $\partial^{2} / \partial x^{2}+\partial^{2} / \partial y^{2}$, and the boundary conditions are assumed periodic for a rectangular region. The solenoidal component of the velocity at each grid point $(x, y)$ is found by

$$
u_{s}(x, y)=-\frac{\partial \psi}{\partial y} v_{s}(x, y)=\frac{\partial \psi}{\partial x} .
$$

The next step is to detect vortices in the field $\left(u_{s}, v_{s}\right)$ and estimate their parameters. Over the last two decades, various approaches have been used to solve this problem in the framework of two-dimensional turbulence. The simplest detection method is to fix some suitable threshold value of the vorticity and associate large values with coherent vortices (McWilliams 1990). Another method is based on the search for stagnation (stationary) elliptic points of the streamfunction, that is, points $(x, y)$ with

$$
\frac{\partial \psi}{\partial x}=\frac{\partial \psi}{\partial y}=0, Q=\left(\frac{\partial^{2} \psi}{\partial x \partial y}\right)^{2}-\frac{\partial^{2} \psi}{\partial x^{2}} \frac{\partial^{2} \psi}{\partial y^{2}}<0 .
$$

Thus, by stagnation point we mean a point where the velocity is 0 . Quantity $Q$ has clear kinematic meaning: if $Q<0$, then the local stream lines are ellipses surrounding the stationary point. It is reported in Benzi et al. (1988) that these two methods give the same results. In Pasquero and Provenzale (2002), a quantity called Okubo-Weiss parameter equivalent to $Q$ is used for general velocity fields to detect the vortex cores. Another set of algorithms is based on wavelets (Schram et al. 2004; Siegel and Weiss 1997; Guan et al. 2003). First, wavelet transform is applied to the vorticity field or the velocity field. The field is then reconstructed through wavelet synthesis to identify its coherent part, and also 
to determine the shape and size of the eddy structures. In all these studies, independent vortices are assumed to be spatially isolated. The algorithms are devised so that only isolated eddies are detected and merging stage is ignored.

The method we develop in this paper is a hybrid one in the sense that both the quantity $Q$ of (4) and a parametric fit for the streamfunction, resembling a wavelet synthesis, are employed. We first detect the centers of eddies considering the Lagrangian paths around each candidate point. Several thresholds are used for this purpose in view of physical considerations. The first threshold is selected at this step; according to our experience with data, a speed of $4 \mathrm{~cm} \mathrm{~s}^{-1}$ or less is considered close to 0 to avoid any skipping of centers readily seen by human eye. To avoid multiple centers close to each other, only one of such candidate centers are retained within a proximity determined by another threshold. Then, we test if the Lagrangian paths around a stagnation point are circles (more generally ellipses) or not (Boyce and DiPrima 2001, 466-467) considering the velocity gradient given by

$$
\boldsymbol{\nabla} V(x, y)=\left[\begin{array}{ll}
\partial u_{s} / \partial x & \partial u_{s} / \partial y \\
\partial v_{s} / \partial x & \partial v_{s} / \partial y
\end{array}\right] .
$$

In view of Eqs. (3) and (4), the determinant of $\nabla V$ is equal to $-Q$. If this determinant is positive at a stagnation point, nearby trajectories form circular or elliptic paths. Hence, we label a stagnation point $(x, y)$ of the velocity field as an eddy center when $\operatorname{det} \nabla V(x, y)$ $>0$.

In the second stage, we follow a parametric approach to estimate the radius and the amplitude of an eddy. The streamfunction is approximated as a sum of essentially Gaussian functions centered on each eddy. The Gaussian function behaves well in the least squares estimation that follows below, in contrast to compact support eddies usually used in the Çinlar model (Çağlar 2000) that create complications in the numerical optimization even if they are as smooth as they can be. On the other hand, functions with compact support are more useful in the modeling phase. Thus, we assume that

$$
\psi(\mathbf{r})=\sum_{j=1}^{n} \psi_{j}(\mathbf{r})
$$

where the contribution $\psi_{j}$ of an eddy centered at $\mathbf{r}_{j}=$ $\left(x_{j}, y_{j}\right)$ to the streamfunction $\psi$, at the point $\mathbf{r}=(x, y)$, is approximated by

$$
\psi_{j}(\mathbf{r})=\tilde{a}_{j} \exp \left\{-\frac{\left|\mathbf{r}-\mathbf{r}_{j}\right|^{2}}{b_{j}^{2}}\right\},
$$

where real $\tilde{a}_{j}$ and $b_{j}>0$ are parameters to be estimated. Then, the vorticity contributed by an eddy centered at $\mathbf{r}_{j}$ is found as

$$
\xi_{j}(\mathbf{r})=\frac{4 \tilde{a}_{j}}{b_{j}^{2}}\left(\frac{\left|\mathbf{r}-\mathbf{r}_{j}\right|^{2}}{b_{j}^{2}}-1\right) \exp \left\{-\frac{\left|\mathbf{r}-\mathbf{r}_{j}\right|^{2}}{b_{j}^{2}}\right\} .
$$

It follows that the eddy centered at $\mathbf{r}_{j}$ generates the velocity field with components given by

$$
\begin{aligned}
& u_{j}(x, y)=\frac{2 \tilde{a}_{j}}{b_{j}} \frac{y-y_{j}}{b_{j}} \exp \left\{-\frac{\left|\mathbf{r}-\mathbf{r}_{j}\right|^{2}}{b_{j}^{2}}\right\}, \\
& v_{j}(x, y)=-\frac{2 \tilde{a}_{j}}{b_{j}} \frac{x-x_{j}}{b_{j}} \exp \left\{-\frac{\left|\mathbf{r}-\mathbf{r}_{j}\right|^{2}}{b_{j}^{2}}\right\} .
\end{aligned}
$$

Hence, we intend to fit the following basic velocity field centered at $(0,0)$ :

$$
u_{0}(x, y)=\frac{y}{r} f(r), v_{0}(x, y)=-\frac{x}{r} f(r),
$$

where $r=|\mathbf{r}|$ and $f(s)=2 s \exp \left(-s^{2}\right)$ for $s \geq 0$.

Then, any other eddy $\left(u_{j}, v_{j}\right)$ is obtained from the basic eddy, as in the stochastic velocity model, by

$$
u_{j}(x, y)=a_{j} u_{0}\left(\frac{\mathbf{r}-\mathbf{r}_{j}}{b_{j}}\right), v_{j}(x, y)=a_{j} v_{0}\left(\frac{\mathbf{r}-\mathbf{r}_{j}}{b_{j}}\right),
$$

where

$$
a=\tilde{a} / b
$$

is the eddy amplitude (intensity) and $b$ is proportional to the eddy radius defined exactly below.

When $n$ eddies are present in a snapshot of data, we can write the streamfunction and vorticity evaluated at center $\mathbf{r}_{i}=\left(x_{i}, y_{i}\right)$ of the eddy $i$ as

$$
\psi\left(\mathbf{r}_{i}\right)=\sum_{j=1}^{n} \psi_{j}\left(\mathbf{r}_{i}\right), \xi\left(\mathbf{r}_{i}\right)=\sum_{j=1}^{n} \xi_{j}\left(\mathbf{r}_{i}\right) i=1, \ldots, n,
$$

which is a nonlinear system of $2 n$ equations in $2 n$ unknowns, namely, $\tilde{a}_{1}, \tilde{a}_{2}, \ldots, \tilde{a}_{n}$, and $b_{1}, b_{2}, \ldots, b_{n}$.

We demonstrate uniqueness of the solution of (8) for $n=2$, namely, for the case of two eddies (see appendix), which is a strong evidence of the solution uniqueness in the general case.

In application, the least squares nonlinear equation solver of MATLAB is employed with the constraints that the radii $b_{i}$ must be in the interval $[0,5 \mathrm{~km}]$ and the amplitude $a_{i}$ can be at most $3.5 \mathrm{~m} \mathrm{~s}^{-1}$ in absolute value. We find a starting solution for the least squares solver by the following argument. If the eddy radii were much less than the distance between them, then

$$
\tilde{a}_{i}=\psi\left(\mathbf{r}_{i}\right), \frac{1}{b_{i}^{2}}=-\frac{\xi\left(\mathbf{r}_{i}\right)}{4 \psi\left(\mathbf{r}_{i}\right)} i=1, \ldots, n
$$


would be good estimators, since they explicitly satisfy the system (8) as can be easily seen from (5) and (6). The solver iterates to find the least squares solution, which stage is computationally intensive, yet feasible, as it is performed only once. At each snapshot, we start with the initial solution (9) to find the amplitudes and the radii. We do not use the solution of the previous snapshot.

To reconcile the numbers between estimation and the modeling phase, we compute the amplitude and radius of an eddy. The Gaussian function does not have a compact support, so one has to decide on the effective radius. Usually, streamfunction or vorticity is used to estimate the radius. Streamfunction contrasts in real data are not so sharp as in model functions, which may or may not be with compact support. We use the streamfunction with a threshold of its $1 / 3$. That is, the radius $r_{0}$ is the point where the peak of $\psi_{0}=\exp \left(-s^{2}\right)$ reduces to its $1 / 3$. Hence, the set of radii are computed as $b_{i} r_{0}$ and the $\tilde{a}_{i}$ are scaled as $\tilde{a}_{i} a_{0}$, where $a_{0}$ is the amplitude of $f$ in the basic eddy given by (7).

We have not imposed any further thresholds because we aim to represent the velocity field at as many grid points as possible. The eddies can overlap, appear to be in the merging state, or possibly be too far away from each other with no restrictions. In other work on vortex analysis (Benzi et al. 1988; McWilliams 1990; Pasquero and Provenzale 2002), the authors tried to identify the area where most of the vorticity is concentrated in the stationary regime as a physical aspect and do not have the aim of representing the velocity field in the transient state. We also target the stationary regime, but in our case, such a regime is not deterministic as in the case of a numerical solution of the vorticity equations, but rather is random. So, vortex mergers can take place or the vortices may exist but not be that obvious to the human eye as a result of the overlap.

\section{Results and discussion}

In this section, estimation results for eddy parameters are reported. In section 5a we present several eddy statistics that are either of common interest or connected to the parameters of the Çinlar model. The parameter estimates themselves are given and discussed in section $5 \mathrm{~b}$. Finally, in section $5 \mathrm{c}$ we validate the Çinlar model by qualitative comparison of synthetic and real velocity fields.

\section{a. Eddy statistics}

The data provide a large sample for the following statistical analysis, namely, a total of $28 \times 24 \times 4=$

\section{(a)}

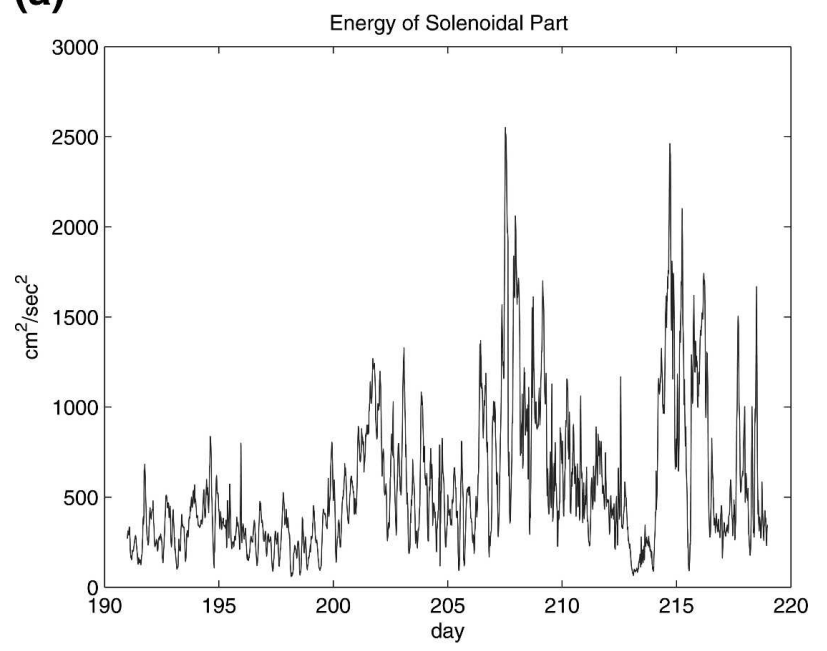

(b)

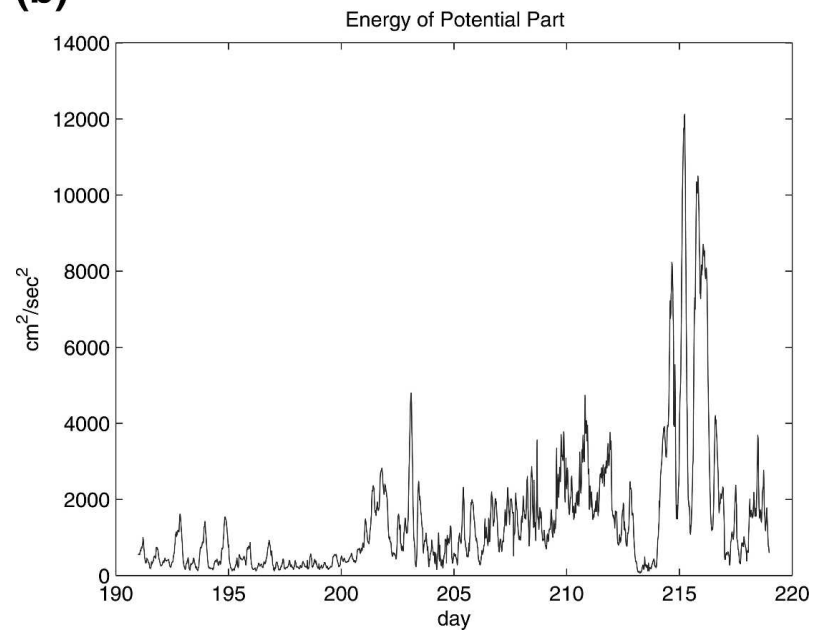

FIG. 2. (a) Average energy of solenoidal velocity. (b) Average energy of potential velocity.

2688 snapshots in time. Since the estimates of eddy amplitudes and radii are recalculated at each time step, they form a time series. After the stationary parts of the time series are detected, all eddies in the snapshots of a stationary piece are sampled together.

Figures 2a,b show the kinetic energy (averaged over the whole region) of the solenoidal and potential parts, respectively, as a function of time. The energy of the solenoidal part is much less than that of the potential part. This is evidence that the velocity structure in the underlying region is essentially three-dimensional. There are two hypotheses for an explanation of these processes. The first is that these eddies originate from the splitting of larger-scale eddies (so-called Tortugas eddies; Fratantoni et al. 1998) that form upstream of the Florida Current (N. Shay and H. Peters 2004, personal communication). Another possibility is that these 
(a)

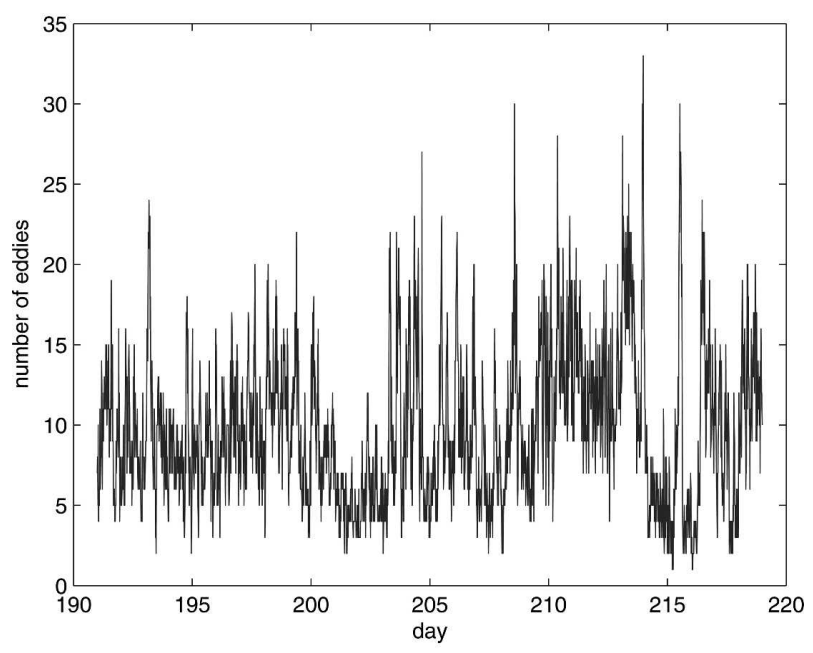

(b)

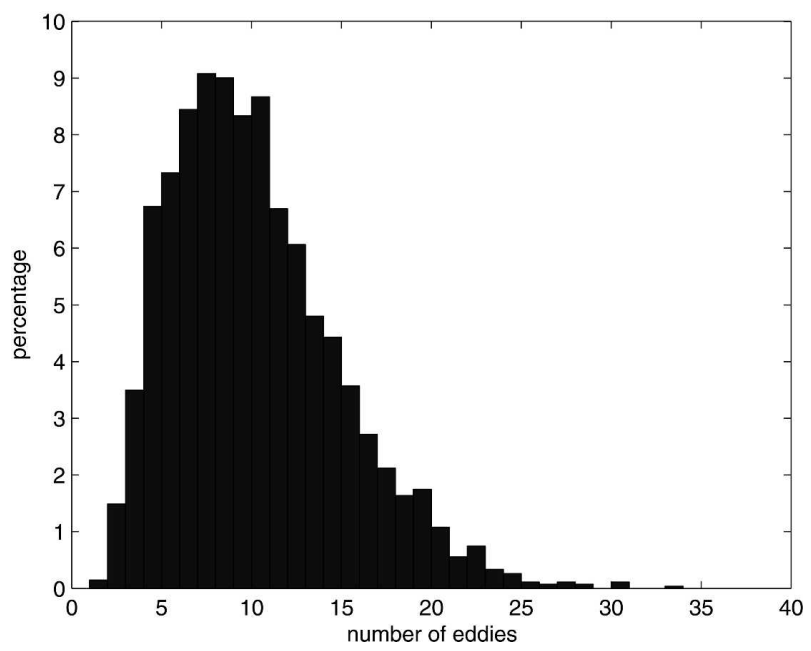

FIG. 3. (a) Time series of the number of eddies. (b) Distribution of the number of eddies per snapshot.

eddies form by the upwelling of Gulf Stream waters (D. Olson 2002, personal communication). More detailed (subsurface and larger domain) observations are needed to pinpoint the exact reason causing these processes. Also, the picture demonstrates clearly that the energy evolution in both cases is nonstationary: the variance in the first period (days 191-204) is certainly lower than that of the second period (days 205-218). This fact determines our strategy in statistical analysis. We first estimate statistics for the whole ensemble (days 191-218) and then elaborate parameter estimates separately for two periods.

The eddy arrival/departure process is illustrated by Fig. 3a plotting the number of eddies versus time moments separated by a 15 -min interval. Notice that this time series looks more or less stationary in contrast to the energy although the extremal number of eddies (25-32) occurs during the second period.

The statistical distribution of the number of eddies per snapshot is shown in Fig. 3b. It is a right skewed distribution with a mean of 9.7 and a standard deviation of 4.7 eddies. Even though the histogram resembles Poisson distribution, it can hardly be identified as Poisson since the latter has equal mean and variance. The similar histograms for the separate periods fit Poisson better (see next subsection).

Figures $4 \mathrm{a}, \mathrm{b}$ clearly demonstrate that the uniform distribution fits well for the distribution of eddy centers. Although any distribution can be associated with eddy centers in the Çinlar model, the uniform distribution has significance because it indicates homogeneous turbulence (E. Çinlar 1994, personal communication). This important special case has been considered extensively in the succeeding studies of the velocity model. Recall that the area in Figs. 4a,b corresponds to that offshore from Fort Lauderdale, Florida, and has a size of approximately $11 \mathrm{~km} \times 11 \mathrm{~km}$.

The distribution of the velocity amplitude (eddy intensity) shown in Fig. 5 is clearly normal with zero mean and a standard deviation of approximately 42.5 $\mathrm{cm} \mathrm{s}^{-1}$. The probability density function of the normal distribution with these parameters is scaled and plotted over the histogram for comparison purposes. The distribution symmetry suggests that there is a balance between cyclones and anticyclones.

In Fig. 6 the distribution of the eddy radii is shown. It is also approximately Gaussian with the mean close to $3 \mathrm{~km}$ except the right-hand-side tail, which demonstrates that probably eddies with a radius of more than $5 \mathrm{~km}$ (which are not identified by our method) affect the estimates. We have imposed the 5-km cutoff for eddy radius due to a limited observation area of 11.25 $\mathrm{km}$ by $11.25 \mathrm{~km}$. On the other hand, one may like to estimate any eddies of size $5 \mathrm{~km}$ or more in view of Fig. 6. We have computed the least squares estimates of the amplitude and radius by removing the respective upper bounds when solving system (8). About $95 \%$ of all eddies have an eddy intensity of $3.5 \mathrm{~m} \mathrm{~s}^{-1}$ or less in absolute value, even when no bounds are imposed. However, the estimation procedure identifies larger and larger eddies as we arbitrarily increase the bound on the radius. Very large radii do not make sense in the limited observation area, but the shape of the distribution could be relevant. The distribution appears skewed to the right in this case. Such a distribution will be considered elsewhere in connection to covariance structures. For the purposes of the current study, we will approximate with a normal distribution. 
(a)

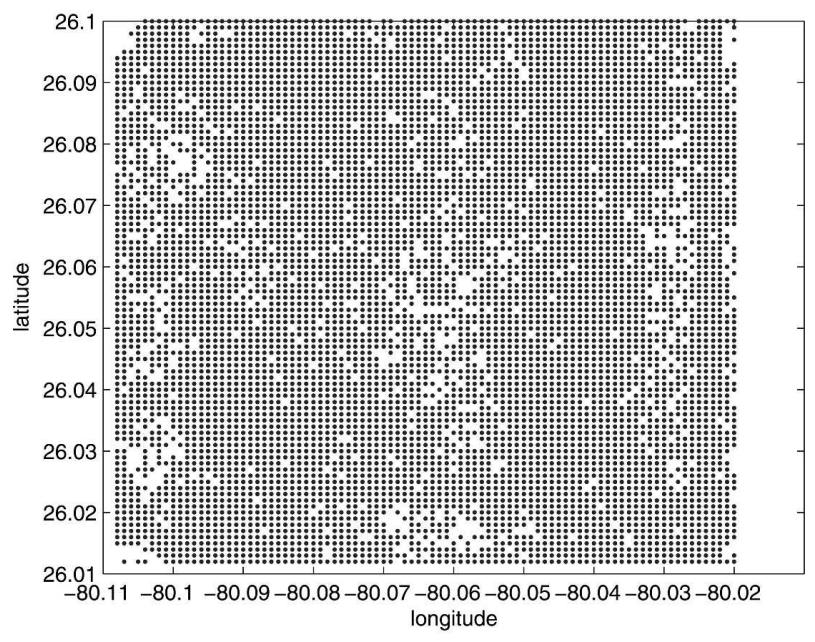

(b)
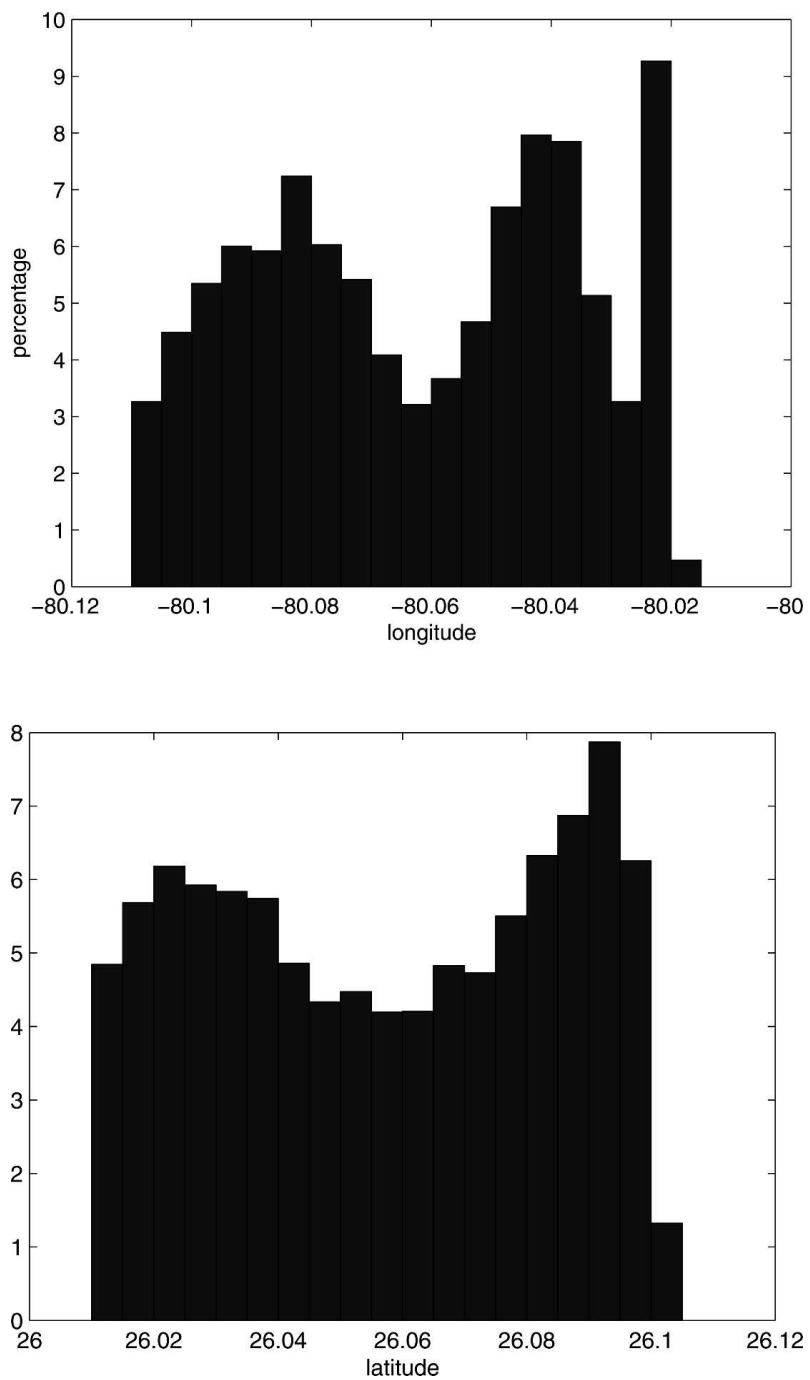

FIG. 4. (a) Scatterplot of eddy centers. (b) Distributions of longitude and latitude of eddy centers.

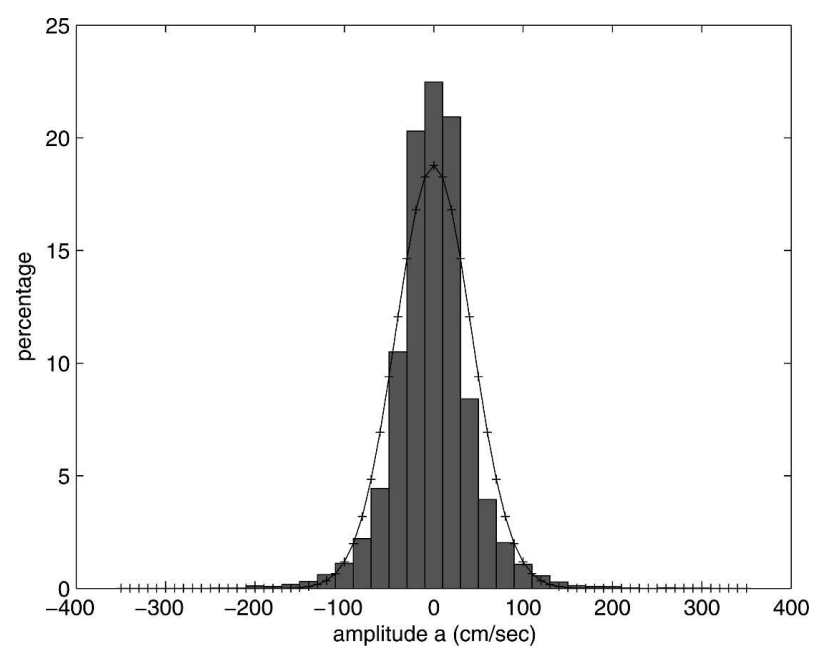

FIG. 5. Distribution of eddy amplitudes.

Figure 7 shows the distribution of lifetime of eddies in the observation window of $11.25 \mathrm{~km} \times 11.25 \mathrm{~km}$ area. Eddies move out of the observation area or just disappear. Accordingly, the lifetime of eddies are exponentially distributed with a mean of approximately $26.5 \mathrm{~min}$ (which is approximately equivalent to three consecutive snapshots). The eddy kinematics will be further considered in connection to the stochastic velocity model in the next subsection where we also describe the computation of the eddy lifetime.

The scatter diagram of amplitude versus radius (Fig. 8a) shows that they are uncorrected. Notice again a clear symmetry in cyclones and anticyclones even for every single eddy size. Independence is not essential in the Çinlar model although it was assumed in simulations given in Çağlar (2000).

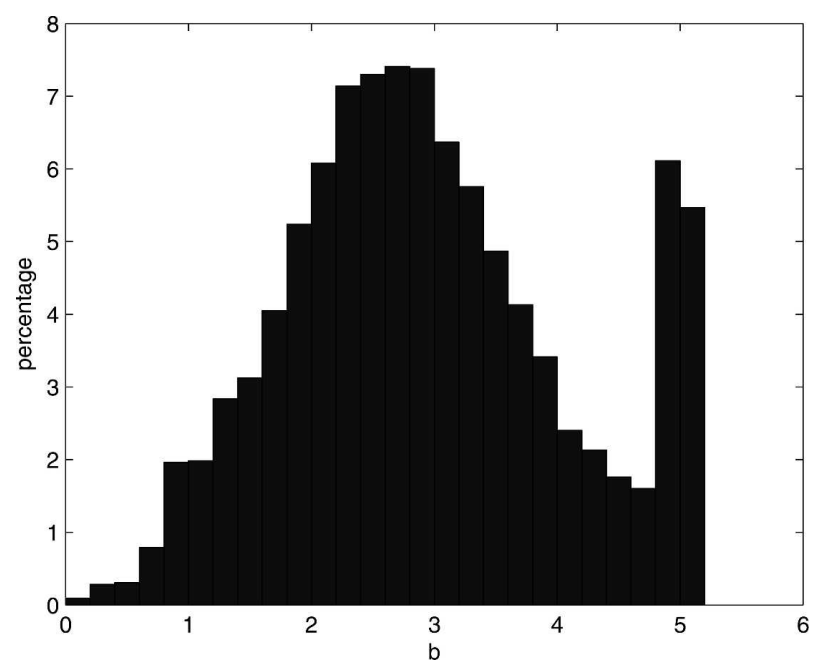

FIG. 6. Distribution of eddy radii. 


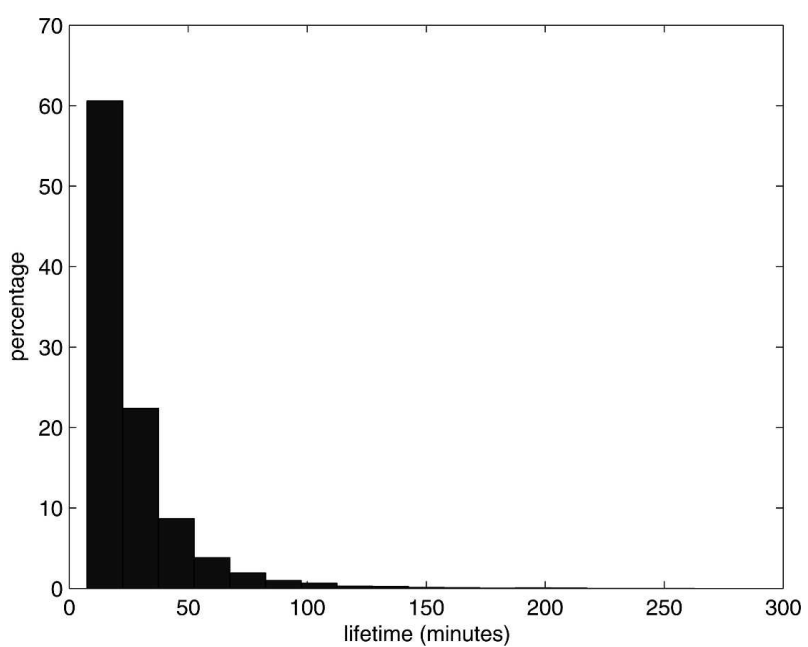

FIG. 7. Distribution of eddy lifetime.

In contrast, the eddy lifetime is clearly related to the size (Fig. 8 b): only eddies of radius of $3.5-4.5 \mathrm{~km}$ can live longer than $3 \mathrm{~h}$ (see the upper-right corner). The observed independence of size and lifetime for shortliving eddies (see the lower part of the diagram) is in agreement with the Çinlar model.

\section{b. Parameter estimates for stochastic velocity model}

In the previous subsection all the detected eddies were treated as coming from the same statistical ensemble in order to get an idea on overall eddy statistics. Here the parameters of the stochastic velocity model will be identified for distinct eddies (defined in the next paragraph) and different time periods for the purpose of being able to synthesize eddy-rich flows.

The distinct eddies, for which the lifetimes have already been reported in Fig. 7, are found by simply searching for nearby eddy centers over successive snapshots of the velocity field and identifying them as identical. For this purpose, we adopt the threshold used earlier for identifying close centers at a single snapshot as explained in section 4. We apply the threshold for comparing the centers at successive snapshots this time. The threshold value is approximately a distance of four grids (i.e., $500 \mathrm{~m}$ ), somewhat lower than the value used earlier for a single snapshot where there are many elliptic points close to each other. We do not distinguish if a vortex merger occurs or the eddy simply vanishes. We have ignored the amplitudes in the identification of distinct eddies, because the estimates of the amplitudes do not show any particular pattern over time. In the complete dataset of snapshots, a total of 26065 eddies have been identified. Of these, $57 \%$ are distinct eddies for which the following parameters can now be estimated:

\section{(a)}

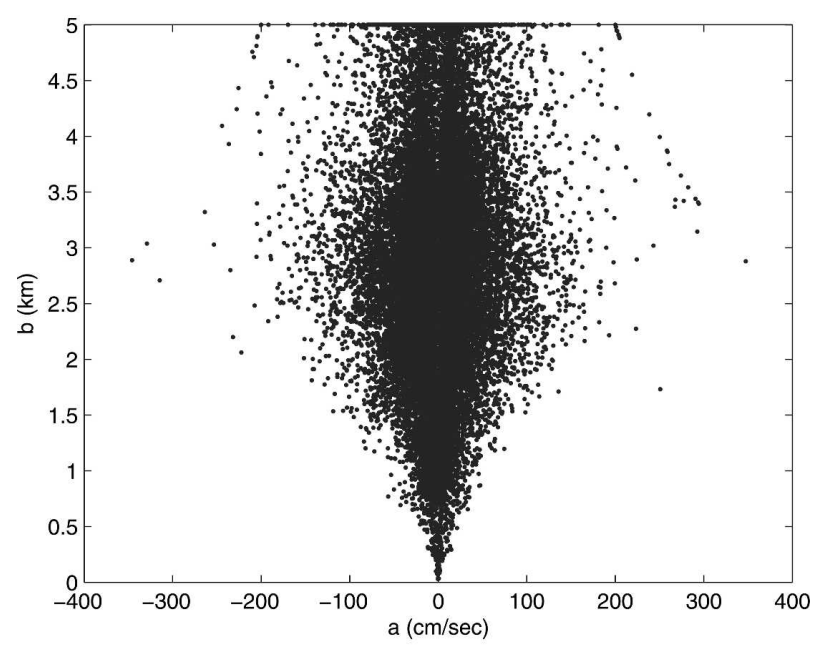

(b)

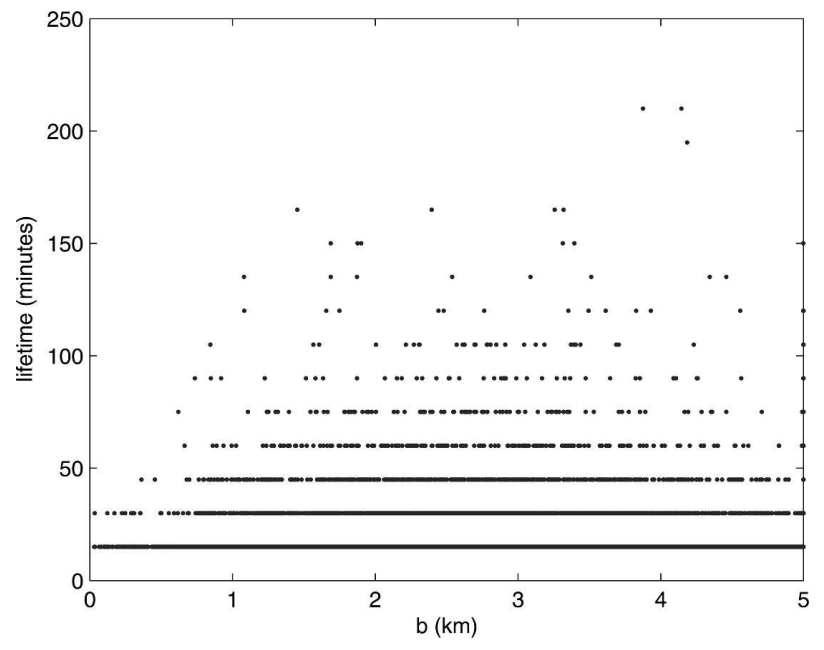

FIG. 8. (a) Scatter diagram of amplitude and radius. (b) Scatter diagram of radius and lifetime.

- the arrival rate $\lambda$;

- the decay rate $c$;

- the distributions of center, amplitude, and radius, respectively.

The eddy arrival process given in Fig. 3a shows the same characteristics of the velocity and the energy time series given in Figs. 1 and 2, respectively: nonstationarity. However, the first half of the observations are stationary. As the exact reason for the creation of such eddies is not known, the explanation of such change in behavior needs to await more extensive observations in this region. A Poisson process fits well to the first part of the sample of arrivals. Namely, we expect the number of arrivals over each 15-min interval to be independent Poisson random variables. The histogram is given 
(a)

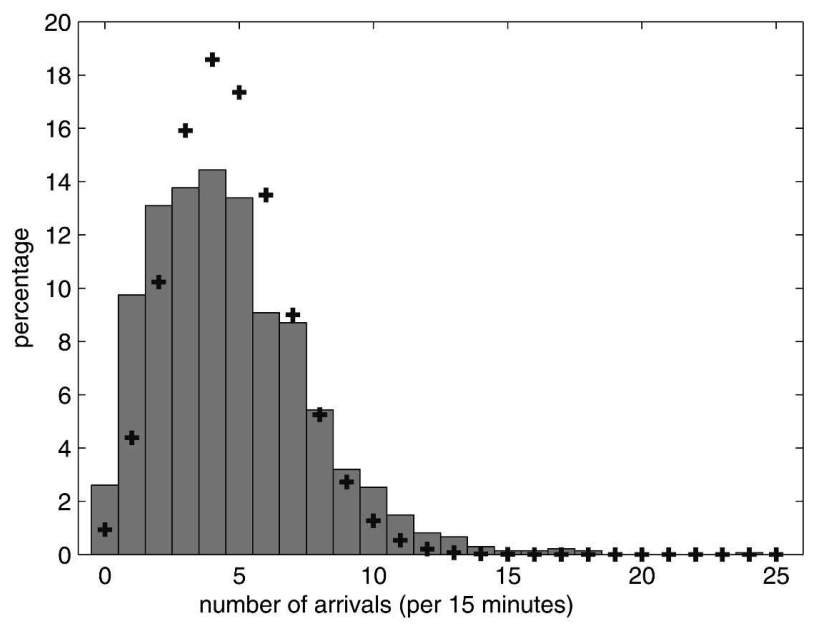

(b)

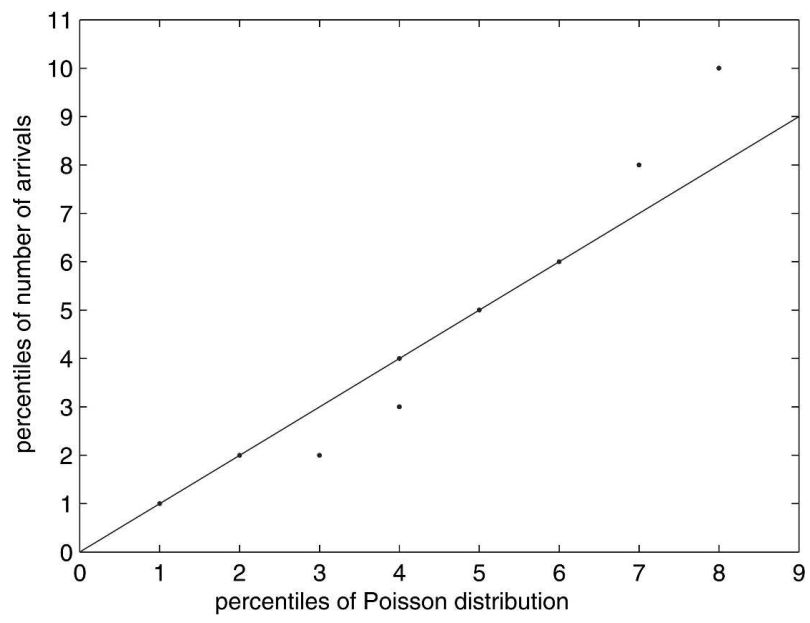

FIG. 9. (a) Distribution and (b) Poisson probability plots during the first period of eddy arrival process.

in Fig. 9a together with a Poisson probability function, and a probability plot is given in Fig. 9b where a good alignment with the $45^{\circ}$ line supports the Poisson hypothesis. If we use the whole time series, which is apparently nonstationary, the fit is not as good. However, it is better than the second half of the arrival process, which we analyze below. The arrival rate in the first part is $\lambda \approx 0.31$ per minute over the complete observation area. Note that the arrival rate is 15 times this value, approximately 4.7 , for each 15 -min time interval given in Fig. 9.

Some of the eddies remain alive for more than one snapshot and so one can talk about the lifetime for them. The eddies extracted from the first time period have the mean lifetime of $28.9 \mathrm{~min}$. According to Little's law in queuing theory of stochastic processes, $L=$ $\lambda W$, where $L$ is the mean number in the system and $W$ is the mean lifetime in the context of this paper. That is, on the average we expect $L=0.31 \times 28.9=8.96$ eddies per snapshot. This estimate of $L$ is in good agreement with another estimate, which can be obtained from the data in Fig. 3b. Indeed, the mean number of eddies per snapshot is 8.99 for the first half of the observations used to form this figure. This agreement supports the threshold used for identifying the nearby eddies in successive snapshots as identical, and hence the estimates of the arrival rate $\lambda$ and the mean lifetime $W$.

We suggest that the eddy arrival process during the second period can also be considered as stationary, but with a higher variance. The Poisson fit and the probability plot are given in Fig. 10. A Poisson process does not fit as good as in the first part of the dataset. The mean of the arrivals in the second part is quite lower than their variance. Therefore, binomial with parameters $n$ and $p$ as another discrete distribution does not fit well either, since its mean $n p$ is larger than its variance $n p(1-p)$. In brief, other efforts are needed to accurately approximate the arrival process in the second half. The arrival rate during this period is estimated as $\lambda \approx 0.42$ per minute over the complete observation area. Note that $\lambda$ being both the mean and variance of Poisson distribution is of higher value than in the first period as expected. The arrival rate is then equal to 6.31 and the mean lifetime is of $24.7 \mathrm{~min}$. As a result, the mean number of eddies per snapshot is found as 10.38 , which is close to 10.40 estimated from the data in Fig. $3 b$.

Poisson arrivals for eddies is the basic assumption in the Çinlar model, which we have shown to be supported by the data. There are sufficiently long time periods where the Poisson process is homogeneous; in other words, the arrival rate $\lambda$ is constant. In the analysis above, we have identified roughly two periods with a low arrival rate and a high arrival rate, respectively.

We have analyzed the distribution of centers, amplitudes, and the radii for distinct eddies for the two regimes of low and high arrivals, separately. Interestingly, the distributions of the centers $\mathbf{z}$ and radii $b$ do not vary over time when analyzed for the first and second half of the time series. Only the amplitude $a$ behaves in two different but intuitive ways. The variance of $a$ in the first half is smaller than the variance in the second half, with standard deviations of 35.2 and $52.4 \mathrm{~cm} \mathrm{~s}^{-1}$, respectively. The distributions are still normal with mean 0 similar to Fig. 5. On the other hand, the distributions of $\mathbf{z}$ and $b$ for distinct eddies are very similar to the distributions given Figs. 4 and 6 with no significant difference in the estimated values of distribution param- 
(a)

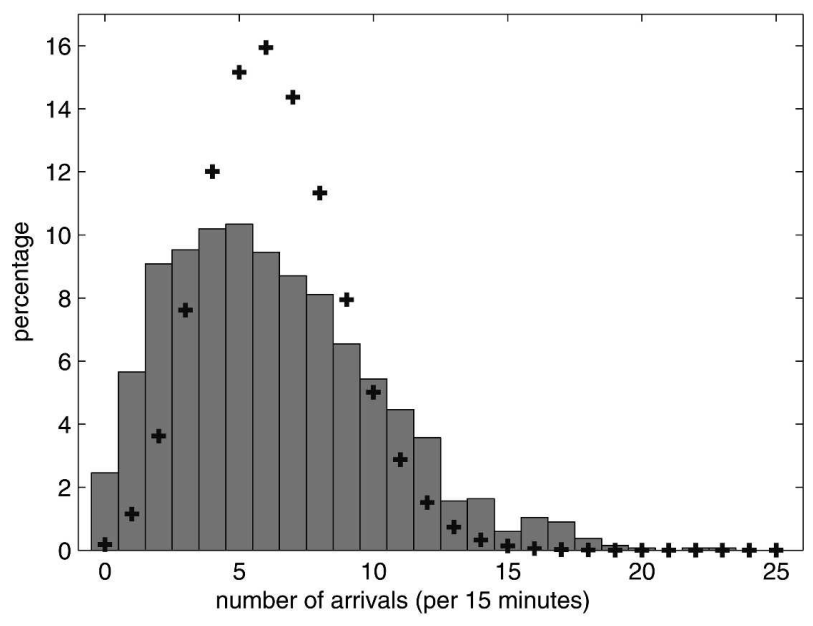

(b)

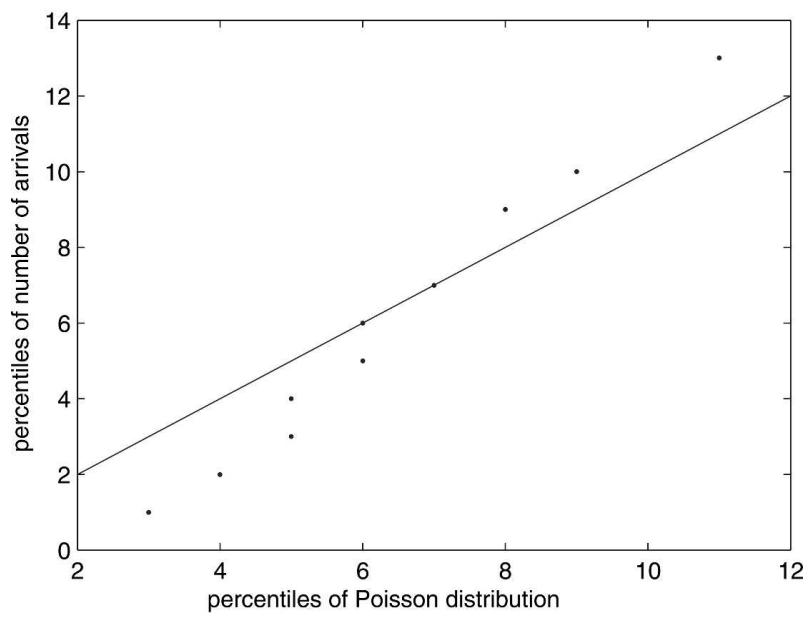

FIG. 10. (a) Distribution and (b) Poisson probability plots during the second period of eddy arrival process.

eters. For example, the radii $b$ are again approximately normally distributed with mean $2.95 \mathrm{~km}$ and standard deviation $1.17 \mathrm{~km}$. When all snapshots are pooled together as in Fig. 6, the mean is again $2.95 \mathrm{~km}$ and the standard deviation is $1.15 \mathrm{~km}$. The centers are uniformly distributed over the whole observation area, which is in good agreement with the Çinlar model for homogeneity in space.

We have studied the kinematics of eddy arrival and disappearance only considering the centers of eddies. In the Çinlar model, the disappearance of eddies is modeled by exponential decay of the amplitude of the eddies so that a stationary velocity field is obtained. The sample of amplitudes for distinct eddies does not show such a streamlined behavior in the dataset. This may be due to the nonperfect estimation procedure, but

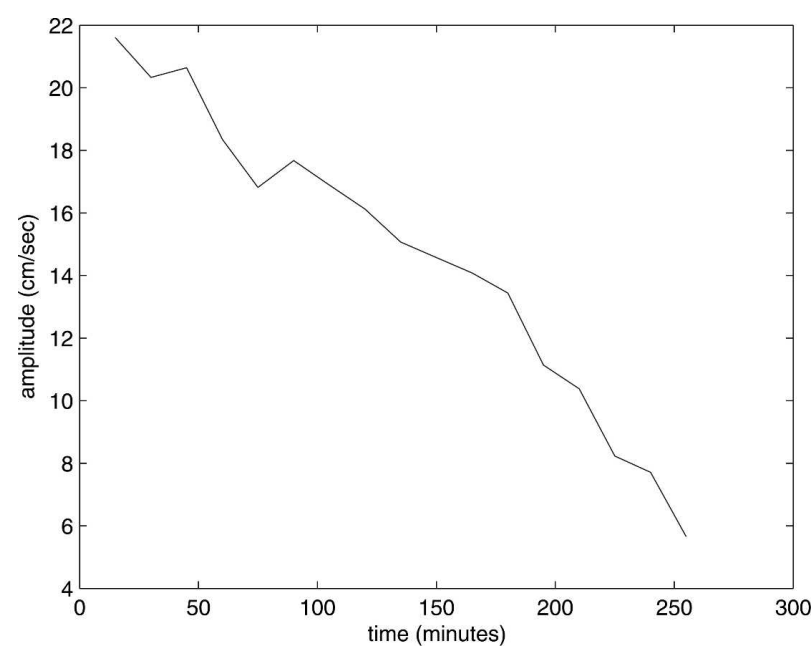

FIG. 11. Mean of the absolute value of the amplitudes vs time.

most probably the real eddy death process is much more complicated than just the exponential decay. Figure 11 shows the time evolution of the mean of the absolute value of the amplitudes. The mean decays linearly, although individual eddies may behave much differently. However, the approximation of decay by exponential law can be useful for obtaining scale estimates and thereby for interpreting the observations. Therefore, we proceed with estimating the value of $c$ as a unique parameter of decay as follows. The typical magnitude of $a$ can be taken equal to its standard deviation as it is a zero mean random variable. In the dataset, we have considered a velocity of $4 \mathrm{~cm} \mathrm{~s}^{-1}$ or less to be close to 0 . As a result,

$$
a \exp (-c t) \approx 4
$$

For example, for the first half of the time series, the lifetime $t$ is $28.9 \mathrm{~min}$ and the magnitude $a$ is $35.2 \mathrm{~cm} \mathrm{~s}^{-1}$ and hence $c \approx 0.08$ per minute in this case. In the second half, the lifetime is smaller and $a$ is larger and a close estimate of $c \approx 0.10$ per minute is obtained consequently. These values are summarized in Table 1as well as the estimates of all other parameters of the model.

An interesting aspect that our stochastic velocity field in fact does not attempt to model is any movement of eddy centers. When identifying two centers to be the same, we have used a threshold value as explained above only over the last two consecutive data snapshots. Hence, it is possible to track the movement of a single eddy from start to end by recording the difference in the coordinates, which happens to be less than the threshold at each time, as we proceed. Figure 12a 
TABLE 1 . Estimates of stochastic velocity model parameters.

\begin{tabular}{lcc}
\hline \hline \multicolumn{1}{c}{ Parameters } & First period & Second period \\
\hline Eddy arrival rate $\lambda$ & $0.31 \mathrm{~min}^{-1}$ & $0.42 \mathrm{~min}^{-1}$ \\
Eddy decay rate $c$ & $0.08 \mathrm{~min}^{-1}$ & $0.10 \mathrm{~min}^{-1}$ \\
Eddy centers $z$ & Uniform over the observation area \\
Eddy velocity & Normal $(0,35.2$ & Normal $(0,52.4$ \\
amplitude $a$ & $\left.\mathrm{~cm} \mathrm{~s}^{-1}\right)$ & $\left.\mathrm{cm} \mathrm{s}^{-1}\right)$ \\
Eddy radius $b$ & Normal $(2.95$, & \\
& $1.17 \mathrm{~km})$ & \\
& & \\
\hline
\end{tabular}

shows a histogram of the movement in longitude direction [west $(-)$-east $(+)]$, and Fig. 12b gives a similar histogram for the latitude direction [south(-)-north(+)]. The unit of number of grids has been used in both figures for comparison purposes. As expected, the centers do not move that far in east-west direction, but some of them move farther toward north. Recalling the threshold value of about four grids for concluding that a center belongs to a single eddy, which is justified above by the statistical expectations of the lifetime and the number of eddies per snapshot as well, we conclude that the centers basically do not move in east-west direction as given in Fig. 12a. The positions are scattered symmetrically around $0(72.4 \%$ is 0$)$ with very low variance and at most a value of 10 grids in both directions. In parallel, the movement toward south is at most 10 grids as well as given in Fig. 12b and most eddies do not move in south-north direction $(68.5 \%$ is 0$)$. However, very few eddies, namely, $0.34 \%$ of all, move farther north more than 10 grids. As a result, our model is expected to capture almost all statistical features of

\section{(a)}

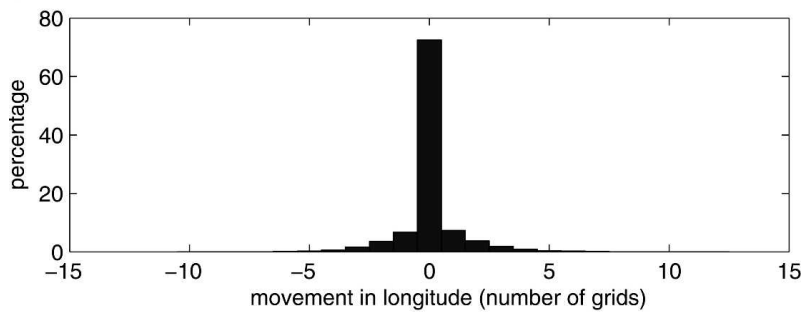

(b)

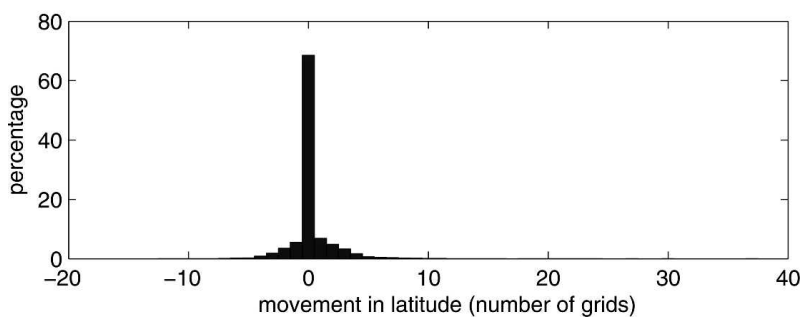

FIG. 12. Distribution of the movement of centers in (a) longitude (b) latitude direction. Unit: number of grids. Grid spacing: $125 \mathrm{~m}$. eddy kinematics via the random appearance of new eddies over the ocean surface and their decay with stationary centers. In particular, the stochastic velocity field $V$ of Eq. (2) satisfies a differential equation in time involving the underlying Poisson point process on time and space (E. Çinlar 1994, personal communication), which leads to an integral representation as given in Çağlar and Çinlar (2001). We aim to utilize this differential equation in future work for integrating the stochastic velocity field to OGCMs as a dynamical parameterization for submesoscale flow.

\section{c. Velocity fields generated by the stochastic velocity model}

There are different approaches to the model validation problem in statistics. One of the popular views is that any quantitative methods such as complete crossvalidation or in-sample error are hardly appropriate for complex nonlinear systems (e.g., Hegger et al. 1999). Instead, the preference should be handed to qualitative methods that are based on visual comparison between the real and synthetic data. An interesting example of such an approach is given in Goff and Jordan (1988) where a stochastic model of seafloor morphology was tested. The submesoscale eddy dynamics is certainly a kind of complex nonlinear process and by this reason we employ the same approach to validate the Çinlar model.

In Fig. 13 two consecutive snapshots from the data are compared with synthetic snapshots coming from the model. We use the parameters of the first period given in Table 1 and the two snapshots are $15 \mathrm{~min}$ apart as in the data. The visual similarity in the field structure is obvious for each snapshot. However, the model evolves in time faster resulting in greater difference between the consecutive snapshots comparatively to the data where the two snapshots are quite similar. It can be related to an unrealistic assumption of the eddy exponential decay discussed before. This difference is dominated visually by the mean flow when added as given in Fig. 14.

\section{Summary and conclusions}

The present study is motivated by recent HF radar observations in the Florida coast, which revealed submesoscale eddies between the Florida Current and the coast. Despite the increasing resolution and realism of OGCMs, such processes are still much smaller than the typical grid spacing, may involve nonhydrostatic dynamics, and are difficult to be captured explicitly at the present time.

The main objective of the present study is first to 
Data
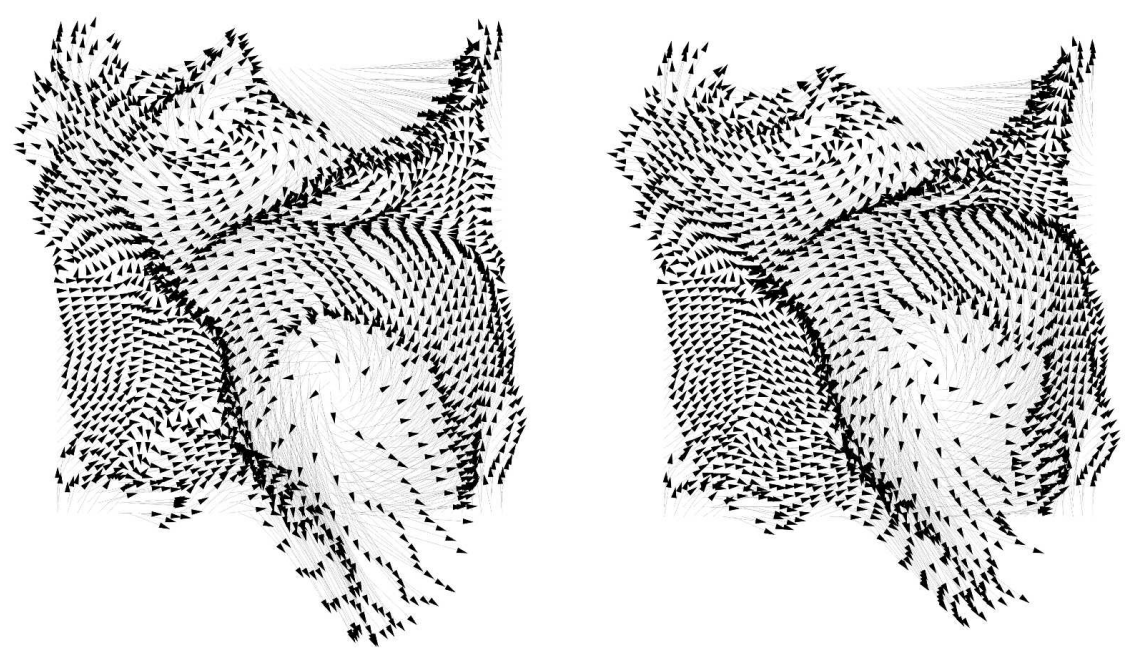

Model
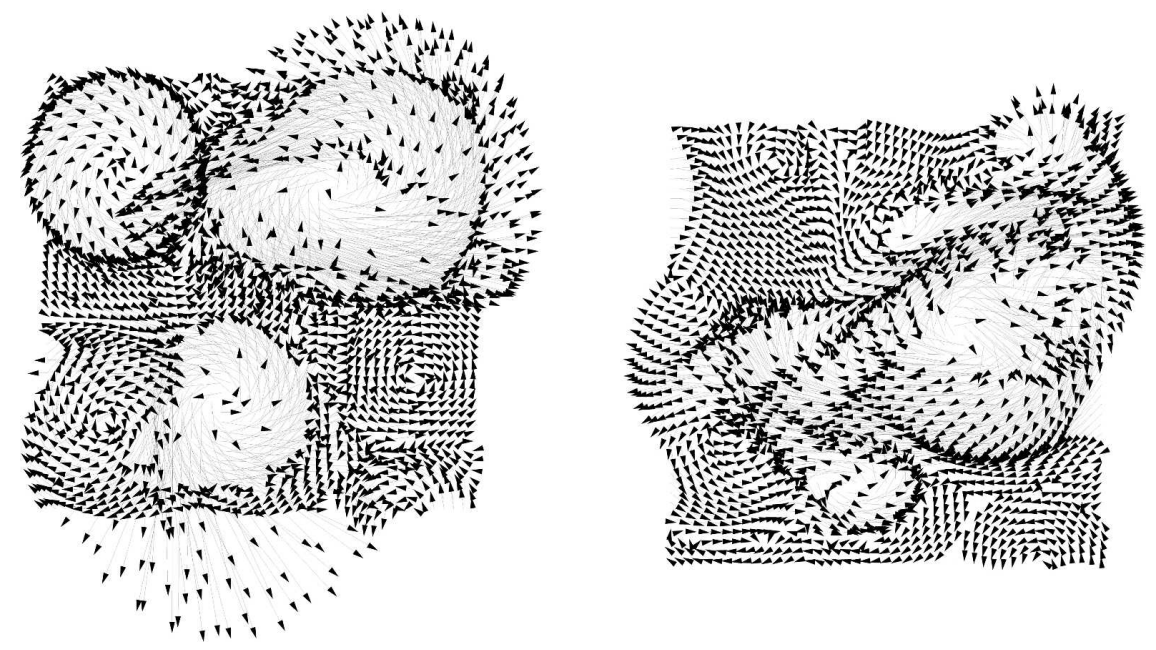

FIG. 13. Snapshots of the velocity field from (top) data and (bottom) stochastic velocity model.

carry out a statistical analysis of the observed velocity field using a new method of detecting eddies and quantifying their characteristics, and then to explore whether the stochastic velocity model put forth by Çağlar and Çinlar (2001) can be employed to parameterize this flow field.

To this end, a statistical analysis of the eddy ensembles extracted from HF radar observations near the Florida coast is performed, which yields an overall view of the distribution of important submesoscale variability characteristics such as the eddy size, amplitude, lifetime, and the number of eddies. An elaborate study for two distinct periods of observations shows that main assumptions behind Çinlar stochastic flow are in fair agreement with the eddy statistics obtained from the data. Moreover, we have validated the Çinlar model by a qualitative comparison of the synthetic and real velocity fields. Therefore, it is found that the Çinlar stochastic velocity field is an adequate tool for parameterization of submesoscale eddy turbulence and could be tried as an adjunct to OGCMs or coastal models in order to reproduce more realistically submesoscale transport processes.

It is also found that not all conditions in the model are supported by data. One deficiency is the eddy amplitude exponential decay that should be revisited in a further development of the model.

One of the important avenues for future research is 
Data
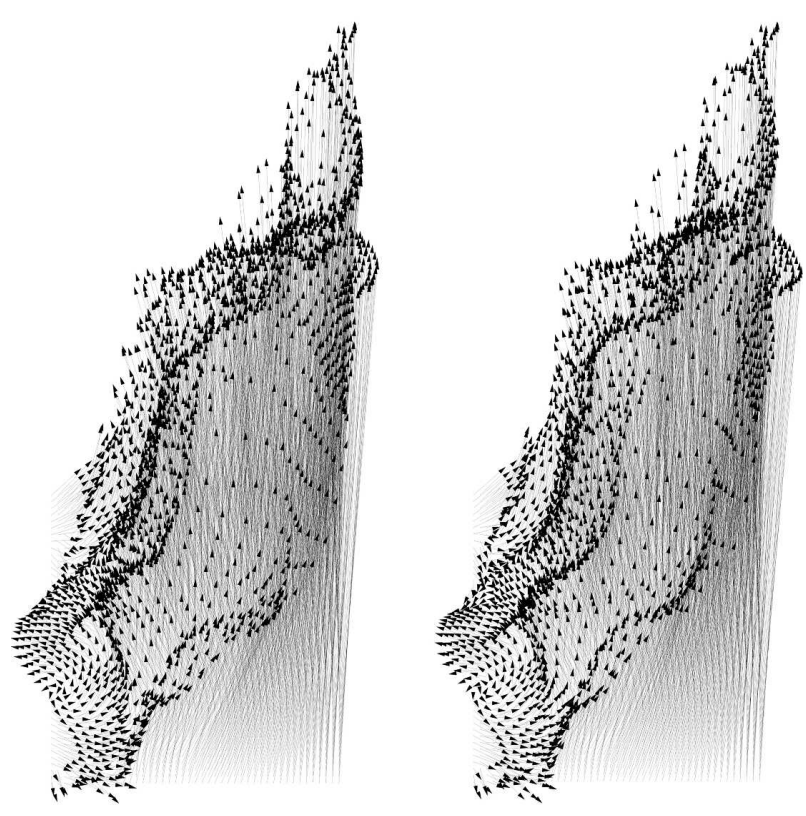

Model
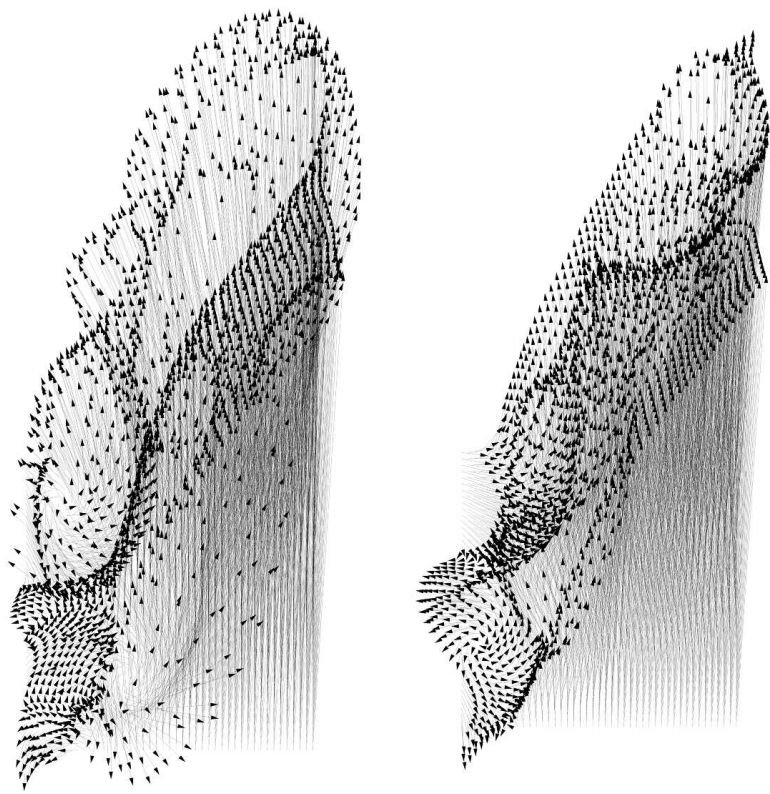

FIG. 14. Snapshots of the velocity field from (top) data and (bottom) model after the mean flow is added.

the application of this stochastic velocity model as a subgrid-scale parameterization of submesoscale processes in larger-scale (coastal and ocean) numerical models. Interesting relevant and ongoing work on the implementation of physically based subgrid-scale models include that by Berloff (2005). For this purpose, the (a)

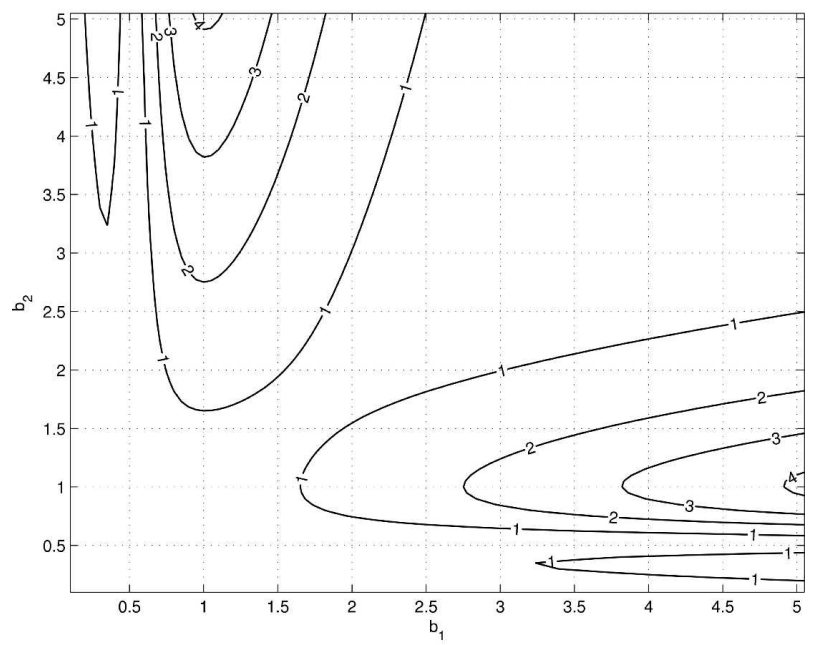

(b)

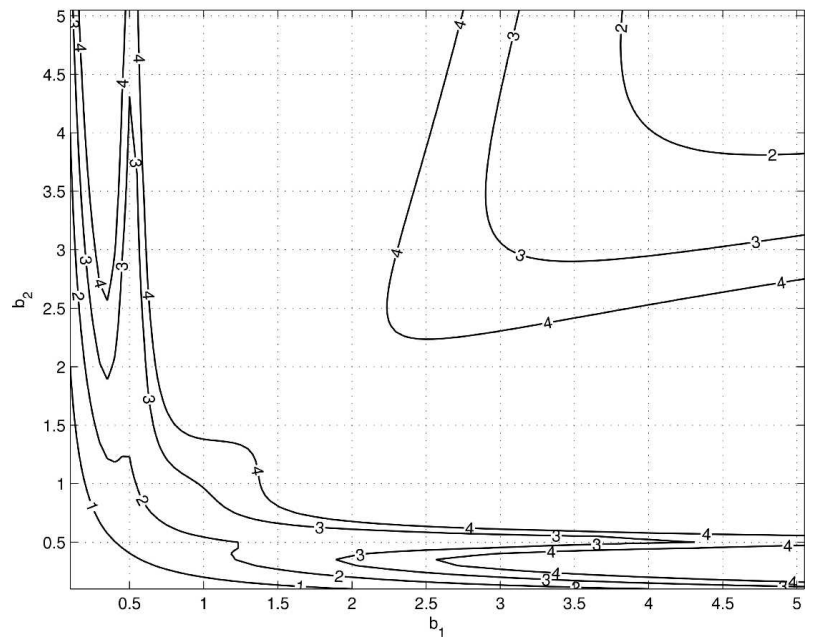

FIG. A1. (a) Contour lines of Jacobian of system (Al) in coordinates $b_{1}, b_{2}$ for $a_{1}=a_{2}$. (b) Same as in (a), but for $a_{1}=5 a_{2}$.

net effect (viz., the stress) exerted by the stochastic velocity field on a scale of the same size as the model grid spacing must be calculated. The analytical nature of the parametric model is convenient to carry out this procedure. An important issue from the perspective of numerical modeling is the dissipative characteristics of the stochastic velocity model, or in more general terms, its impact on the energy cascade. These issues will be topics of further research.

Acknowledgments. This work is supported by NSF Grants OISE-352448, OISE-352637, TUBITAK-NSF Grant 103Y147, and by ONR Grants N00014-99-0042 (L. Piterbarg) and N00014-05-10095 (T. Özgökmen). The authors thank L. Shay for many useful discussions 
about the HF-radar data, and A. Mariano and E. Ryan for making the data available. We are also grateful to anonymous reviewers for their constructive comments.

\section{APPENDIX}

\section{On the Uniqueness of Solutions of (8)}

System (8) for $n=2$ in variables $x_{i}=\tilde{a}_{i}, y_{i}=d^{2} / b_{i}^{2}$, $i=1,2$ is written as

$$
\begin{aligned}
x_{1}+x_{2} f\left(y_{1}\right) & =s_{1} \\
x_{1} f\left(y_{1}\right)+x_{2} & =s_{2} \\
x_{1} g\left(y_{1}\right)+x_{2} h\left(y_{2}\right) & =v_{1} \\
x_{1} h\left(y_{1}\right)+x_{2} g\left(y_{2}\right) & =v_{2},
\end{aligned}
$$

where $f(y)=\exp (-y), g(y)=-y, h(y)=\left(y^{2}-y\right)$ $\exp (-y)$, and $s_{1}, s_{2}, v_{1}, v_{2}$ are arbitrary observed values of the streamfunction and vorticity, respectively. Let $J$ $=J\left(x_{1}, x_{2}, y_{1}, y_{2}\right)$ be the Jacobian of (Al), which can be written in explicit form. The expression is relatively lengthy and we do not expose it. Direct computations show that $J>0.2$ for a wide range of $0.01<x_{1}, x_{2}, y_{1}$, $y_{2}<100$. This is illustrated by the contour lines of $J$ as function of eddy radii $b_{1}$ and $b_{2}$ in the case of eddies with equal amplitudes $\left(a_{1}=a_{2}\right.$; Fig. A1, upper panel) and in the case of sharply different amplitudes $\left(a_{1}=\right.$ $5 a_{2}$, Fig. A1 lower panel). Radii $b_{1}$ and $b_{2}$ are given in the units of the distance between the eddies.

\section{REFERENCES}

Benzi, R., S. Patarnello, and P. Santangelo, 1988: Self-similar coherent structures in two-dimensional decaying turbulence. $J$. Phys. A: Math. Gen., 21, 1221-1237.

Berloff, P. S., 2005: Random-forcing model of mesoscale oceanic eddies. J. Fluid Mech., 529, 71-95.

Boyce, W. E., and R. C. DiPrima, 2001: Elementary Differential Equations and Boundary Value Problems. 7th ed. Wiley, 768 pp.

Çağlar, M., 2000: Simulation of homogeneous and incompressible Çinlar flows. Appl. Math. Model., 24, 297-314.

, 2003: Dispersion of mass by two-dimensional homogeneous and incompressible Çinlar flows. Appl. Math. Model., 27, 997-1011.
— noise velocity fields. Stochastic Processes Appl., 94, 29-49.

Fratantoni, P. S., T. N. Lee, G. P. Podesta, and F. Muller-Karger, 1998: The influence of loop current perturbations on the formation and evolution of Tortugas eddies in the southern Straits of Florida. J. Geophys. Res., 103 (C11), 24 759-24 779.

Goff, J. A., and T. H. Jordan, 1988: Stochastic modeling of seafloor morphology: Inversion of sea beam data for secondorder statistics. J. Geophys. Res., 93 (B11), 13 589-13 608.

Guan, S., C. H. Lai, and G. W. Wei, 2003: Characterizing the spatiotemporal dynamics of turbulence. Comput. Phys. Commun., 155, 77-91.

Hegger, R., H. Kantz, and T. Schreiber, 1999: Practical implementation of non-linear time series methods: The TISEAN package. Chaos, 9, 413-435.

Mariano, A. J., T. M. Chin, and T. M. Özgökmen, 2003: Stochastic boundary conditions for coastal flow modeling. Geophys. Res. Lett., 30, 1457, doi:10.1029/2003GL016972.

McWilliams, J. C., 1990: The vortices of two-dimensional turbulence. J. Fluid Mech., 219, 361-385.

Pasquero, C., and A. Provenzale, 2002: Vortex statistics from Eulerian and Lagrangian time series. Phys. Rev. Lett., 89, 284501, doi:10.1103/PhysRevLett.89.284501.

Peters, H., L. Shay, A. Mariano, and T. Cook, 2002: Current variability on a narrow shelf with large ambient vorticity. J. Geophys. Res., 107, 3087, doi:10.1029/2001JC000813.

Schram, C., P. Rambaud, and M. L. Riethmuller, 2004: Wavelet based eddy structure eduction from a backward facing step flow investigated using particle image velocimetry. Exp. Fluids, 36, 233-245.

Shay, L., and Coauthors, 2000: VHF radar detects oceanic submesoscale vortex along Florida coast. Eos, Trans. Amer. Geophys. Union, 81, 209-213.

— T. Took, H. Peters, A. Mariano, R. Weisberg, P. An, A. Soloviev, and M. Luther, 2002: Very high-frequency radar mapping of surface currents. IEEE J. Oceanic Eng., 27, 155169.

Siegel, A., and J. B. Weiss, 1997: A wavelet packet census algorithm for calculating vortex statistics. Phys. Fluids, 9, 19881999.

Soloviev, A., M. Luther, and R. H. Weisberg, 2003a: Energetic baroclinic super-tidal oscillations on the shelf off southeast Florida. Geophys. Res. Lett., 30, 1463, doi:10.1029/ 2002GL016603.

—, R. J. Walker, R. H. Weisberg, and M. E. Luther, 2003b: Coastal observatory investigates energetic current oscillations on southeast Florida shelf. Eos, Trans. Amer. Geophys. Union, 84, 441.

Zirbel, C. L., and E. Çinlar, 1996: Dispersion of particle systems in Brownian flows. Adv. Appl. Probab., 28, 53-74. 